\title{
An evaluation model and tool for Defence capability options
}

\author{
$\underline{\text { M.-T. Nguyen }}^{\mathrm{a}}$, A. Coutts ${ }^{\mathrm{a}}$, J. Knoll ${ }^{\mathrm{a}}$, M. Zuparic ${ }^{\mathrm{a}}$, J. Peacock ${ }^{\mathrm{a}}$ ，D. Bilusich ${ }^{\mathrm{a}}$, M. Galister ${ }^{\mathrm{a}}$ \& K. Johns \\ ${ }^{a}$ Joint \& Operations Analysis Division, Defence Science and Technology Group, Australia \\ Email: \{Minh-Tuan.Nguyen|Andrew.Coutts|Julia.Knoll|Mathew.Zuparic\}adst.defence.gov.au
}

\begin{abstract}
The 2020 Force Structure Plan (FSP) sets out adjustments to Defence capability investments and will provide options to deliver the new strategic objectives outlined in the 2020 Defence Strategic Update ${ }^{1}$. This plan is developed and reviewed within a four year Defence Capability Assessment Program (DCAP) cycle. The first two years of the cycle delivers an update to Defence on force structure in response to changes in strategic circumstances and policy, while the full cycle delivers a revised FSP. Defence Science \& Technology Group (DSTG) have proposed an evaluation approach by integrating various methodologies: Bayesian network (BN), multi-criteria decision making (MCDM), Monte-Carlo simulation with parallel computing and multi-objective evolutionary algorithms (MOEA). By working with Force Design Division (FDD) (within the Vice Chief of the Defence Force (VCDF) Executive), who are responsible for delivering the FSP, we have developed a Bayesian Reasoning Value Model (BRVM) and a tool for managing and analysing data collected from subject matter experts (SMEs). An evaluation of Defence capability options is then conducted via this Bayesian Network Analysis Tool for Force Structure Plan (BNAT-FSP).

Interactions among the force structure components (Defence element, force package, operational effect and mission) are defined by logical or physical links which complicate the evaluation of the dependability of these force structure models. In addition, the different operational scenarios and threats present a challenge to decision makers (DMs) in providing a clear answer to the problem of determining the value of proposed capability options. BN is employed to establish a qualitative and quantitative representation of the relations between the variables of the model considered and calculate standard values of uncertain capabilities (e.g. mission success, operation/force effectiveness, e tc). MCDM is designed to combine the influence of multiple BN models built from different sc enarios/missions. With our BRVM reaching the size of thousands of variables, exact inference in BNs is apparently infeasible. We apply a stochastic sampling method together with Monte-Carlo simulation, which is run on parallel virtual computers, for reaching evaluation results at our desired accuracy. MOEA is also used in our sensitivity analysis to find the optimal level of mission success and the impact level of each contributing force.

The proposed multi-method approach provides modelling and data collection structures that assist in making explicit the force structure, scenario parameters, and the DMs' preferences. This significantly reduced the time-consuming elicitation process, and helped us to deliver the option evaluation results on time at a desired accuracy.
\end{abstract}

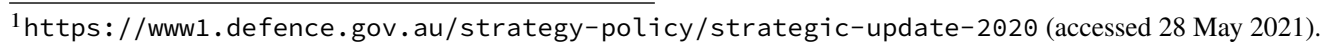

Keywords: Bayesian network, BN, multi-criteria decision making, MCDM, analytic hierarchy process, AHP 


\section{INTRODUCTION}

The 2020 Force Structure Plan (FSP) [Department of Defence 2020] sets out adjustments to Defence capability investments and will provide options to deliver the new strategic objectives outlined in the 2020 Defence Strategic Update to shape Australia's strategic environment, deter actions against Australia's interests, and if required, respond with credible military force. This plan is developed and reviewed within a four year Defence Capability Assessment Program (DCAP) cycle. The first two years of the cycle delivers an update to Defence on force structure in response to changes in strategic circumstances and policy, while the full cycle delivers a revised FSP.

Interactions among the force structure components (Defence element, force package, operational effect and mission) are defined by logical or physical links which complicate the evaluation of the dependability of these force structure models. In addition, the different operational scenarios and threats present a challenge to decision makers (DMs) in providing a clear answer to the problem of determining the value of proposed capability options ${ }^{2}$ These challenging requirements have forced us to consider a novel approach that differs greatly from the previous FSP study [Peacock et al. 2019].

This paper integrates various methodologies: Bayesian network (BN), multi-criteria decision making (MCDM), Monte-Carlo simulation with parallel computing, and multi-objective evolutionary algorithms (MOEA) to present a Bayesian Reasoning Value Model (BRVM) and an analysis tool for evaluating Defence capability options. BN is employed to establish a qualitative and quantitative representation of the relations between the variables of the model considered and calculate standard values of uncertain capabilities (e.g. mission success, operation/force effectiveness, etc.). MCDM is designed to combine the influence of multiple BN models built from different scenarios/missions. With our BRVM reaching the size of thousands of variables, exact inference in BNs is infeasible. We apply a stochastic sampling method together with Monte-Carlo simulation, which is run on parallel virtual computers, for reaching evaluation results at our desired accuracy. MOEA is also used in our sensitivity analysis to find the optimal level of mission success and the impact level of each contributing force.

In order to manage data collected from subject matter experts (SMEs) and to analyse a very large number of model outputs from all possible contributing forces, a prototype tool of the FSP model, named Bayesian Network Analysis Tool for Force Structure Plan (BNAT-FSP), has been developed to perform inferences over the BN, to evaluate the current force structure, to examine proposed capability options using data analysis, visualisation and sensitivity analysis techniques. The tool is 'packaged' with BN software SMILE (Structural Modelling, Inference, and Learning Engine) [Druzdzel 1999], a built-in MCDM framework, and a Monte-Carlo experiment setting to allow required calculations to be completely automated and results to be captured in both tabular and graphical forms.

The capability option evaluation is a continuation of the study on the BN modelling [Cao and Chau 2015; Nguyen and Cao 2017; Nguyen and Cao 2019] and the development of its accompanied decision support tool [Nguyen, Cao, and Chau 2016|. The work presented here represents a much larger-scale problem with a thousand BN nodes and links and a decision support tool development for dealing with the evaluation accuracy when using stochastic sampling method in $\mathrm{BN}$ inferences.

\section{DEFENCE CAPABILITY OPTIONS - DECISION PROBLEM}

The purpose of an evaluation framework is to select capability options at the desired operational effectiveness so the required mission can be accomplished. The measurement of each capability option is determined by force structure/allocation that provides operational effects for achieving various conditions and leading to required mission success. It is therefore desirable to develop a model to support upcoming decisions for prioritising the capability options.

Let's denote $D C \stackrel{\text { def }}{=}\left\{D C_{i}, \quad i=1, \ldots, n\right\}$ the set of $n$ decisive conditions (DCs) that allows commanders to gain a marked advantage over an opponent or contribute materially to achieve mission objectives [NATO 2010]. Force packages (FPs) represent a grouping of related lower level capabilities (known as Defence Elements). Each capability is assigned a level of operational readiness

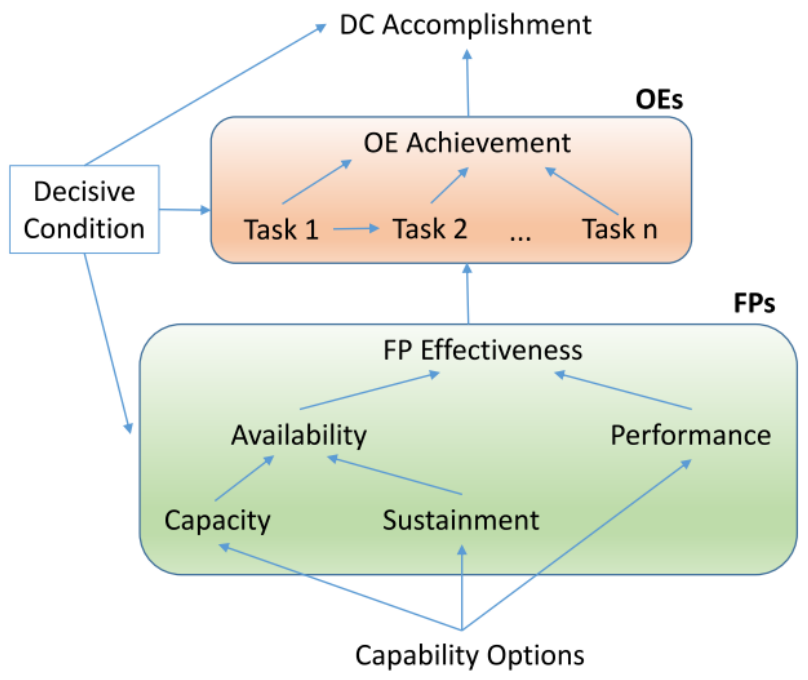

Figure 1. Dependencies between model components

\footnotetext{
${ }^{2}$ Capability options refer to proposed changes to capability (systems and/or personnel) that directly impact Defence Elements and Force Packages in terms of availability and performance.
} 
and depends on the availability of trained personnel, the availability of major platforms, combat systems and supplies, and the standard of collective training [Gaidow, Boey, and Egudo 2006]. A process for converting armed force resources into fighting power is called operational effects (OEs) [Millett, Murray, and Watman 1986]. These OEs will contribute and establish the required $\mathrm{DC}^{3}$. We restrict attention to the following three effectiveness levels in our proposed model: FP-OE-DC. We will name this proposed model in the next section as the BRVM. The dependencies between model components are summarised in Figure 1.

The capability evaluation process can be divided into two steps: the first step is to take each capability option (CO) and measure which FPs will be altered/enhanced from the status quo ('do nothing' option); and the second is to assess its cascade effect from FPs to OEs and then DCs. In our illustrated example, we consider 200 options in a model structure of 6 DCs, 13 OEs and 62 FPs; and have to prioritise these COs.

Let $U_{i j}$ be the utility function of the DC accomplishment for $D C_{i}, i=1, \ldots, n$ when capability option $O_{j}, j=1, \ldots, m$ is selected (i.e. fully funded). The prioritisation list of capability options is thus established based on the evaluation results of $\left\{U_{i j}\right\}, \forall i=1, \ldots, n ; \forall j=1, \ldots, m$.

\section{CAPABILITy OPTION VAlUes - Hybrid eVAluation MODEL}

We have observed and learned from the previous FSP study [Peacock et al. 2019] that using MCDM alone is not applicable to our evaluation framework because the problem is large and complex, in particular containing a number of uncertainty situations caused by incomplete or noisy information. Furthermore, it doesn't model inter-dependencies among DCs, OEs and FPs in the considered force structures. Decision making can be improved if the interrelations among these elements are explicitly modelled and reasoned with rather than ignored by some unrealistic assumptions.

In order to define and evaluate a capability option value function, $V_{j} \stackrel{\text { def }}{=} f\left(U_{1 j}, U_{2 j}, \ldots, U_{n j}\right)$, we utilise both BN and MCDM methodologies, and present a hybrid model for evaluating the operational impact of different capability options. We first build a BN for the force structure (FP-OE - DC) model (hence named BRVM). The utility value of the DC accomplishment $\left\{U_{i j}\right\}$ is obtained by inferring from this BN.

A MCDM framework is then adopted to integrate the influence of various DC utilities $U_{i j}$ and to finally calculate the capability option value $V_{j}$. MCDM evaluates the influence of multiple criteria by calculating a utility value where each criterion takes a certain weight. The pairwise comparison weighting method based on the analytic hierarchy process (AHP) [Saaty 1980] is used in this work and implemented in our decision support tool BNAT-FSP (Section 4).

The $\mathrm{BN}$ is a graphical model for conceptualising and quantifying uncertainty about the causal relationships between variables and consists of

- a list of nodes representing random variables $\mathrm{X} \stackrel{\text { def }}{=}\left\{X_{1}, X_{2}, \ldots, X_{k}\right\}$

- a list of directed links expressing causal probabilistic relationships between the variables

- conditional probability tables (CPTs) $\left\{P\left(X_{i} \mid \Pi_{i}\right)\right\}$ detailing the strength of causal relationship between the nodes, where $\Pi_{i} \subseteq \mathrm{X} \backslash\left\{X_{i}\right\}$ is the parent node set of $X_{i}$.

Using Bayes' theorem, the joint probability distribution in the $\mathrm{BN}$ can be calculated by the multiplication of the conditional probabilities of all the nodes [Pearl 1988] and written as follow:

$$
p\left(X_{1}, X_{2}, \ldots, X_{k}\right)=\prod_{i=1}^{k} p\left(X_{i} \mid \Pi_{i}\right)
$$

Establishment of the BN model requires three components: (1) build the network structure - set up the network nodes and links; (2) determine the network parameters - populate the CPTs; and (3) carry out probability propagation network inference, i.e. calculating the probability of each node given evidences.

We have used the BN package GeNI $4^{4}$ and SMILE [Druzdzel 1999] to create the force structure BRVM. GeNIe provides a good graphical interface for designing and developing a network structure and also has various tools for its CPTs to be populated. GeNIe allows modularity in large and complex systems such as BRVM by using the GeNIe special node type 'submodel'. The sub-model hosts sub-graphs of the entire graph and its structure can be examined in separation from the entire model. All FP and OE nodes in the typical FP-OE-DC network 5 shown in Figure 2 are 'submodel' types:

- FP sub-model produces probability to effectively achieve its assigned roles under various threats.

- OE sub-model produces probability to successfully conduct relevant operational tasks and create superior effects that contribute to achieving DC objectives.

${ }^{3}$ DCs represent key phases or sections of a higher level mission and as such have their own set of objectives and requirements for specific OEs. ${ }^{4}$ http: //support.bayesfusion.com/docs/ (accessed 9 October 2017).

${ }^{5}$ Note that the $\mathrm{BN}$ evaluation framework presented here do not represent an agreed or endorsed position by Defence. 


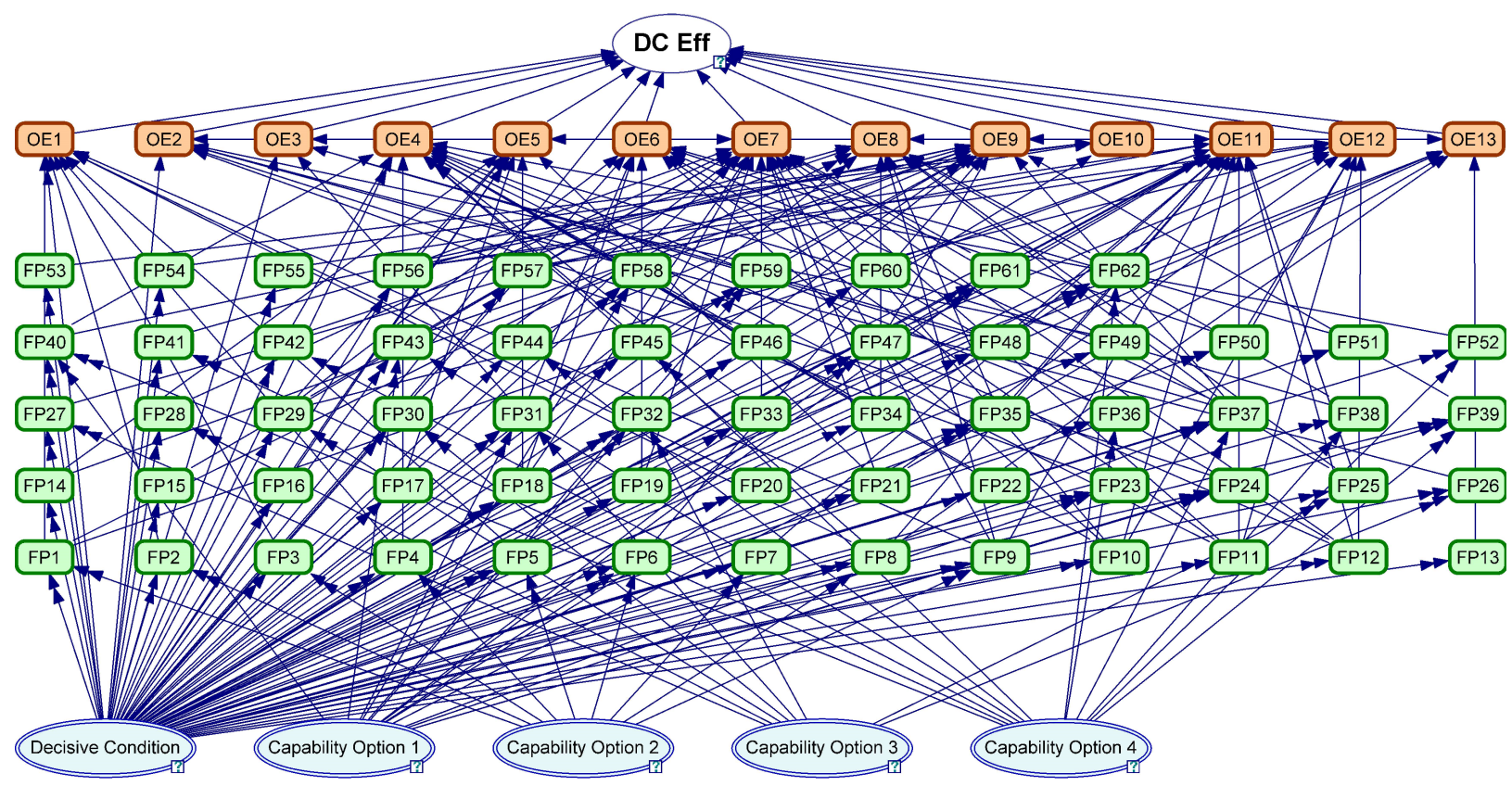

Figure 2. An illustrative example for modelling the force structure (FP-OE-DC) using BN

As our BRVM is large and complex with many interdependencies required at the OE levels, building models manually with GeNIe is a time-consuming process. We have therefore used the SMILE package with our built-in tools for creating BRVM [Knoll et al.2021], while GeNIe is used for visualisation and final adjustment if needed.

The capability option value functions are then defined by additive multi-attribute utility functions [Keeney and Raiffa 1976] and expected utility functions [von Neumann and Morgenstern 1953]

$$
V_{j} \stackrel{\text { def }}{=} \sum_{i=1}^{n} w_{i} U_{i j}, \text { and } U_{i j} \stackrel{\text { def }}{=} \sum_{k=1}^{s} p_{i j}^{k} U_{i j}^{k}, \forall j=1, \ldots, m
$$

where

- there is $s$ number of states/outcomes for the 'DC Eff' node of DC $i$ and Option $j$;

- $\left\{w_{i} \mid \sum_{i=1}^{n} w_{i}=1\right\}$ is weighting constant sets derived from MCDM process;

- $\left\{p_{k}^{i j} \mid \sum_{k=1}^{s} p_{k}^{i j}=1\right\}$ is probability distribution for the 'DC Eff' node; and

- $U_{i j}^{k}$ is the utility value of DC $i$ and Option $j$ in State $k, k=1, \ldots, s$.

In our illustrative example, we show 4 options in a model structure of 13 OEs and 62 FPs. However, we have tested in our work with a larger number of FPs and few hundred options by directly manipulating the impact of each option to the performance, capacity and sustainment

Objects in the network:
\begin{tabular}{|l|r|r|l|}
\hline Object & Count & States & Parameters / Independent \\
\hline Nodes & 348 & 700 & $206606 / 103305$ \\
\hline Chance-General & 343 & 686 & $206592 / 103296$ \\
Deterministic & 5 & 14 & $14 / 9$ \\
Arcs & 767 & & \\
\hline Submodels & 75 & & \\
\hline
\end{tabular}

Figure 3. BN properties displayed by GeNIe nodes in the relevant FP sub-models (see Figure 1). This feature is implemented in our decision support tool, BNAT, to deal with much bigger BN models in reality. Notwithstanding this, the above BN has 343 'chance' nodes (whose relationship with its parents is probabilistic) with 103,296 independent parameters (representing probability of every combination of states of model variables) to be elicited from subject matter experts (SMEs) for populating its CPTs (see BN information in Figure 3). It is clearly infeasible to obtain all of the required data points individually. We thus apply the ACE (Application for Conditional probability Elicitation) method proposed in Hassall et al. 2019 and also extend it to cover both independent and dependent effects in our parameter elicitation process [Johns, Nguyen, and Galister 2021]. 


\section{BAYESIAN NETWORK ANALYSIS TOOL SUITE}

We modify the BNAT [Nguyen 2020] developed for our previous land combat vehicle project [Nguyen and Cao 2019]. This application allows end-users to easily perform inferences over the $\mathrm{BN}$ as well as visualisation and comparison of results. BNAT is a Microsoft Excel workbook 'packaged' with public software allowing the required calculations to be completely automated and results to be captured in both tabular and graphical forms.

\subsection{Cause-and-effect tracing}

Documenting and linking possible explanations of the evaluation results to original collected data assists with the transparency of the proposed framework and model. The cause-and-effect tracing function in BNAT allows us to select any displayed result, and to track down and find its contributing factors with SMEs' quantitative and qualitative reasoning. One can see and compare quickly any issue between effective and allocated forces. Typical examples for a low achievement of OEs is illustrated in Figure 4 In this illustrative example, 'oe13_task1', 'oe13_task2' and 'oe4' are the main cause for 'oe13' low effectiveness. However, the low effectiveness of 'oe1' appears to result from a high threat with unsuitable forces. Detailed reasoning can be retrieved by looking at the original data captured from SMEs and its CPT generation from the associated hyperlinks.
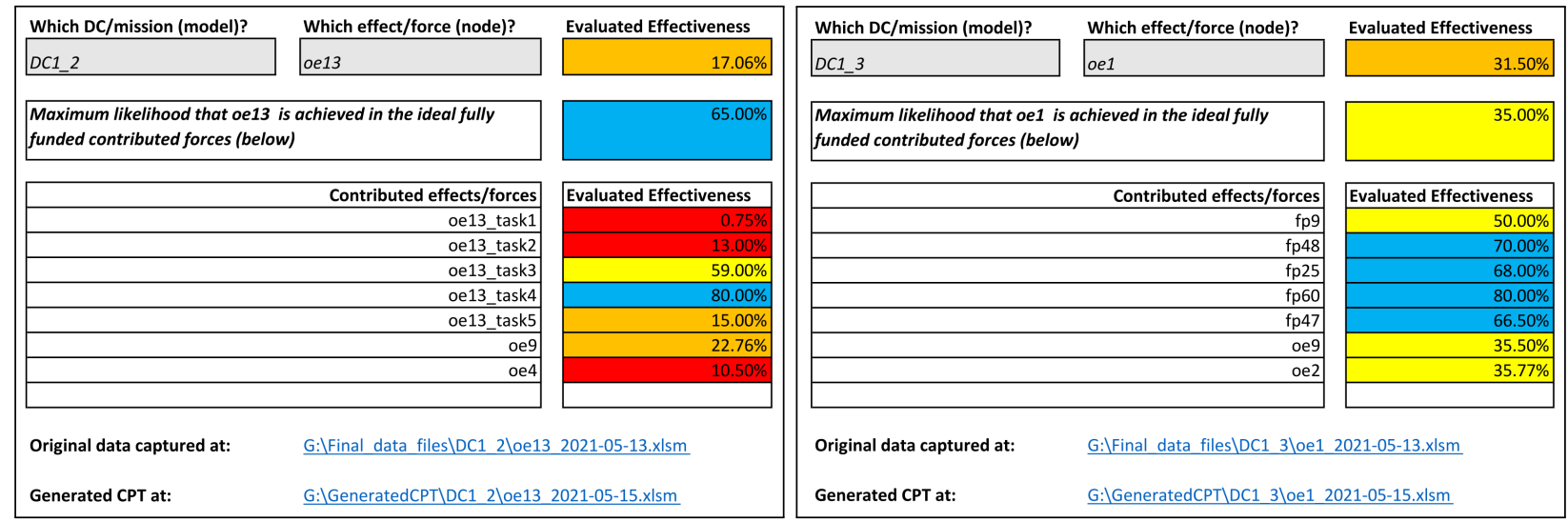

Figure 4. Force effectiveness versus force allocation/threat (for OE13 (left) and OE1 (right))

\subsection{Sensitivity analysis}

Two levels in our BRVM can be tested using BNAT for its sensitivity to the DC accomplishment or OE achievement by varying 'FP Effectiveness' or its parents' 'Performance' and 'Availability' from $\pm 10 \%$ to $\pm 60 \%$ with $10 \%$ increment. One can use this analysis to determine an appropriate level of investment in the required capability. For example, there will be no benefit beyond a $40 \%$ increase in FP8 effectiveness as shown in Figure 5 We can also use the sensitivity analysis results to compile a top 10 FPs of operational or force allocation risk [Zuparic, Coutts, and Peacock 2021].

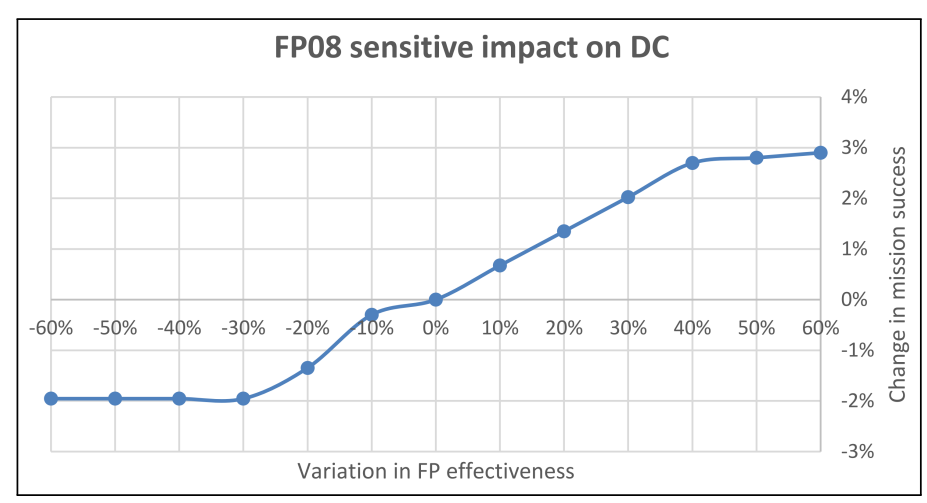

Figure 5. Typical FP sensitivity result

\subsection{Multi-objective optimisation}

Sensitivity allows us to see the impact of changes to a single FP on the force structure (Figure 5). This does not allow us to consider the optimum configuration across multiple possible changes. Multi-objective optimisation using the concept of Pareto optimality ${ }^{6}$ will find a combined impact of all force factors to achieve the required DC/OEs at its 'best' level. A MOEA framework [Hadka 2014] with the non-dominated sorting genetic algorithm II (NSGA-II) [Deb et al.2002] is deployed in the tool for finding optimal levels for each FP. Figure 6 shows us at least 7 'Pareto optimal' possibilities for maximising the effectiveness of the considered DC, OE1, OE2, OE8, OE9 and OE137.

\footnotetext{
${ }^{6}$ A solution is said to be Pareto (non-dominated) when there is no other solution in the space which is better with regards to all decision variables.

${ }^{7}$ Data presented here is fictitious and used for illustrative purposes only.
} 


\begin{tabular}{|c|c|}
\hline Input & Fixed or Free \\
\hline FP1 & Free \\
\hline FP2 & Free \\
\hline FP3 & Free \\
\hline FP4 & Free \\
\hline FP5 & Free \\
\hline FP6 & Fixed \\
\hline FP7 & Free \\
\hline FP8 & Free \\
\hline FP9 & Free \\
\hline FP10 & Fixed \\
\hline FP11 & Free \\
\hline FP12 & Free \\
\hline FP13 & Free \\
\hline FP14 & Free \\
\hline FP15 & Free \\
\hline
\end{tabular}

\section{Searching 'Best' Options}

\begin{tabular}{|l|c|}
\hline \multicolumn{2}{|l|}{ Maximising Effectiveness } \\
\hline Decisive Condition & Yes \\
\hline OE1 & Yes \\
\hline OE2 & Yes \\
\hline OE3 & No \\
\hline OE4 & No \\
\hline OE5 & No \\
\hline OE6 & No \\
\hline OE7 & No \\
\hline OE8 & Yes \\
\hline OE9 & Yes \\
\hline OE10 & No \\
\hline OE11 & No \\
\hline OE12 & No \\
\hline OE13 & Yes \\
\hline
\end{tabular}

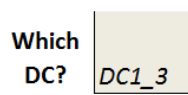

\begin{tabular}{|r|r|r|r|r|r|r|}
\hline Option 1 & Option 2 & Option 3 & Option 4 & Option 5 & Option 6 & Option 7 \\
\hline $65.20 \%$ & $65.20 \%$ & $65.17 \%$ & $65.13 \%$ & $65.10 \%$ & $65.06 \%$ & $65.03 \%$ \\
\hline $68.67 \%$ & $68.67 \%$ & $68.67 \%$ & $68.67 \%$ & $68.67 \%$ & $68.67 \%$ & $68.67 \%$ \\
\hline $30.69 \%$ & $30.69 \%$ & $30.51 \%$ & $30.33 \%$ & $30.15 \%$ & $29.97 \%$ & $29.80 \%$ \\
\hline & & & & & & \\
\hline & & & & & & \\
\hline & & & & & & \\
\hline & & & & & & \\
\hline & & & & & & \\
\hline $24.00 \%$ & $24.00 \%$ & $24.00 \%$ & $24.00 \%$ & $24.00 \%$ & $24.00 \%$ & $24.00 \%$ \\
\hline $70.59 \%$ & $70.59 \%$ & $69.84 \%$ & $69.09 \%$ & $68.34 \%$ & $67.59 \%$ & $66.83 \%$ \\
\hline & & & & & & \\
\hline & & & & & & \\
\hline & & & & & & \\
\hline $62.50 \%$ & $62.50 \%$ & $62.50 \%$ & $62.50 \%$ & $62.51 \%$ & $62.51 \%$ & $62.51 \%$ \\
\hline & & & & & & \\
\hline
\end{tabular}

Figure 6. Illustrative example of interactive force selection with MOEA

\subsection{Capability option ranking}

One of BNAT's main functions is to produce the ranking of the proposed COs. Each $\mathrm{CO}$ will produce an increased/decreased level of FP performance and availability. BNAT will then input these changes inside the BRVM and carry out a BN inference again to get the probability distribution for the 'DC Eff' node. An AHP session with senior SMEs is also organised to elicit the weights among the considered DCs and the CO value $V_{j}$ is finally calculated (see Equation 2). We also compare $V_{j}$ and risk/impact factors for validating the correct behaviour of our BRVM (see [Zuparic, Coutts, and Peacock 2021] for more detail).

\subsection{Numerical experiments}

Our BRVM is very large and complex, particularly in the OE sub-models. GeNIe and SMILE with the default clustering (exact) algorithm [Lauritzen and Spiegelhalter 1988] fails to run a Bayesian inference on some nodes in our BRVM. We thus switched to a stochastic sampling algorithm. One of the best available algorithms that is also implemented in SMILE for discrete Bayesian networks, is the estimated posterior importance sampling (EPIS) [Yuan and Druzdzel[2006]. The evaluation of each option was produced within a second on a PC i7-4770 CPU @ $3.4 \mathrm{GHz}$ with $16 \mathrm{~GB}$ of RAM using the default setting (1,000 generated samples). In order to obtain our desired accuracy in our option prioritisation list, we applied Monte-Carlo simulation with 1000 replications at an increased sample size of 1,000,000. Approximately 100 hours is needed to run with this setup. However, parallel computing on 10 virtual machines reduced the execution time to less than 10 hours 8 .

\section{Conclusion}

This paper illustrates an integration of BN, MCDM, MOEA and Monte-Carlo experiments for evaluating Defence capability options.

The proposed multi-method approach provides modelling and data collection structures that assist in making explicit the force structure, scenario parameters, and the DMs' preferences. This significantly reduced the time-consuming elicitation process, and helped us to deliver the option evaluation results on time at a desired accuracy. In addition, a tool was provided for automating, documenting and verifying some of our evaluation processes. The BRVM and BNAT-FSP tools allow a quick what-if capability, for example producing a sensitivity analysis on certain FPs from any investment, or investigating the impact of various FP performance or availability changes on the required missions.

\section{ACKNOWLeDgments}

The authors would like to acknowledge Force Design Division (FDD) for supporting our project and providing subject matter experts in various workshops. Special thanks go to our colleagues in Land Capability Analysis branch and Strategy \& Joint Force branch for their advice on mathematical modelling aspects of our proposed model and analysis tool.

${ }^{8}$ See Nguyen, Coutts, and Zuparic 2021 for all BNAT features and how to set up and use it. 
M.-T. Nguyen et al., An evaluation model and tool for Defence capability options

\section{REFERENCES}

Cao, T. and Chau, W. (2015) "Bayesian Network Model for Analysing Operational Impact of Land Combat Vehicle". In: the 12th Engineering Mathematics and Applications Conference (EMAC). Adelaide, Australia.

Deb, K., Pratap, A., Agarwal, S., and Meyarivan, T. (2002) “A fast and elitist multiobjective genetic algorithm: NSGAII". In: IEEE Transactions on Evolutionary Computation 6 (2), 182-197.

Department of Defence (June 2020) 2020 Force Structure Plan. Ed. by Commonwealth of Australia.

Druzdzel, M. J. (1999) "SMILE: Structural Modeling, Inference, and Learning Engine and GeNIe: A Development Environment for Graphical Decision-theoretic Models". In: Proceedings of the 16th National Conference on Artificial Intelligence, AAAI '99/IAAI '99. Orlando, Florida, USA, 902-903. ISBN: 0-262-51106-1.

Gaidow, S., Boey, S., and Egudo, R. (2006) "A Review of the Capability Options Development \& Analysis System and the Role of Risk Management”. (DSTO-GD-0473), Defence Science and Technology Organisation, Australia.

Hadka, D. (2014) MOEA Framework User Guide: A Free and Open Source Java Framework for Multiobjective Optimization (Version 2.4). [online] http: / /www. moeaframework.org/(accesed May 2018).

Hassall, K. L., Dailey, G., Zawadzka, J., Milne, A. E., Harris, J. A., Corstanje, R., and Whitmore, A. P. (2019) "Facilitating the elicitation of beliefs for use in Bayesian Belief modelling". In: Environmental Modelling \& Software 122, 104539. ISSN: 1364-8152.

Johns, K., Nguyen, M.-T., and Galister, M. (2021) “Bayesian network model parameter tool for force structure plan: A user's guide". In preparation for publication in Defence Science and Technology Report Series, Australia.

Keeney, R. and Raiffa, H. (1976) Decisions with Multiple Objectives: Performances and Value Trade-Offs. Wiley. New York.

Knoll, J., Nguyen, M.-T., Bilusich, D., and Peacock, J. (2021) “Bayesian network model building tool for force structure plan: A user's guide”. In preparation for publication in Defence Science and Technology Report Series, Australia.

Lauritzen, S. L. and Spiegelhalter, D. J. (1988) "Local Computations with Probabilities on Graphical Structures and Their Application to Expert Systems". In: Journal of the Royal Statistical Society. Series B (Methodological) 50 (2), 157-224. ISSN: 00359246. uRL: http://www.jstor.org/stable/2345762.

Millett, A. R., Murray, W., and Watman, K. H. (1986) “The Effectiveness of Military Organizations”. In: International Security 11 (1), 37-71. ISSN: 01622889, 15314804. uRL: http: / /www.jstor .org/stable/2538875

NATO (Dec. 21, 2010) Allied joint doctrine AJP-01(D). Ed. by Allied Joint Publication, North atlantic treaty organization.

von Neumann, J. and Morgenstern, O. (1953) Theory of Games and Economic Behavior. 3rd. Princeton University Press. New York.

Nguyen, M.-T. (2020) “Bayesian Network Analysis Tool for Evaluating Land Combat Vehicle System: A User's Guide”. (DST-Group-GD-1069), Defence Science and Technology Group, Australia.

Nguyen, M.-T. and Cao, T. (2017) “A hybrid decision making model for evaluating land combat vehicle system”. In: 22nd International Congress on Modelling and Simulation, MODSIM2017. Ed. by Syme, G., Hatton MacDonald, D., Fulton, B., and Piantadosi, J. Hobart, Australia, 1399-1405. IsBn: 978-0-9872143-7-9.

- (2019) "A Multi-Method Approach to Evaluate Land Combat Vehicle System". In: Int. J. Applied Decision Sciences 12 (4), 337-360.

Nguyen, M.-T., Cao, T., and Chau, W. (2016) "Bayesian Network Analysis Tool for Land Combat Vehicle System Evaluation". In: the 24th National Conference of the Australian Society for Operations Research (ASOR). Canberra, Australia.

Nguyen, M.-T., Coutts, A., and Zuparic, M. (2021) "Bayesian network analysis tool suite for evaluating capability options: A user's guide". Submitted for publication in Defence Science and Technology Report Series, Australia.

Peacock, J., Blumson, D., Mangalasinghe, J., Hepworth, A., Coutts, A., and Lo, E. H. S. (2019) "Baselining the Wholeof-Force Capability and Capacity of the Australian Defence Force". In: 23rd International Congress on Modelling and Simulation, MODSIM2019. Ed. by Elsawah, S. Canberra, Australia, 249-255. ISBN: 978-0-9758400-9-2.

Pearl, J. (1988) Probabilistic Reasoning in Intelligent Systems. Morgan Kaufmann. San Francisco.

Saaty, T. L. (1980) The Analytic Hierarchy Process. McGraw-Hill. New York.

Yuan, C. and Druzdzel, M. J. (2006) "Importance sampling algorithms for Bayesian networks: Principles and performance". In: Mathematical and Computer Modelling 43 (9). Optimization and Control for Military Applications, 1189-1207. IssN: 0895-7177. Dor: https : / / doi . org/10 . 1016/ j . mcm . 2005.05.020 URL: https: //www.sciencedirect.com/science/article/pi i/S0895717705005443.

Zuparic, M., Coutts, A., and Peacock, J. (2021) "Validation and analysis of the Bayesian reasoning value model". In preparation for publication in Defence Science and Technology Report Series, Australia. 